\title{
Carfentrazone-ethyl, Isolado e Associado a Duas Formulações De Glyphosate no Controle de DuAs Espécies de Trapoeraba ${ }^{1}$
}

\author{
Carfentrazone-ethyl Isolated and in Mixture with Two Glyphosate Formulations on the Control \\ of Two Dayflower Species
}

\begin{abstract}
RONCHI, C.P. ${ }^{2}$, SILVA. A.A. ${ }^{3}$, FERREIRA, L.R. ${ }^{3}$, MIRANDA, G.V. ${ }^{3}$ e TERRA, A.A. ${ }^{4}$
RESUMO - Esta pesquisa teve como objetivo avaliar a eficácia do herbicida carfentrazoneethyl, isolado ou associado ao glyphosate e ao glyphosate potássico, no controle de duas espécies de plantas daninhas conhecidas como trapoeraba: Commelina diffusa e Commelina benghalensis. Para isso, segmentos de caule dessas plantas foram transplantados e submetidos a crescimento em vasos que continham $12 \mathrm{~L}$ de substrato, durante 120 dias. Os experimentos (um por espécie de trapoeraba) foram conduzidos no delineamento experimental em blocos casualizados, com quatro repetições, sendo constituídos de carfentrazone-ethyl nas doses de $0,10,20,30$, 40 e $50 \mathrm{~g} \mathrm{ha}^{-1}$, isoladas ou aplicadas em mistura com o glyphosate e o glyphosate potássico, ambos na dose de $720 \mathrm{~g} \mathrm{ha}^{-1}$. Foram feitas avaliações de controle e da biomassa fresca da parte aérea (BFPA). C. diffusa foi mais tolerante ao carfentrazone-ethyl e à sua mistura ao glyphosate e ao glyphosate potássico do que $C$. benghalensis. Tanto o glyphosate quanto o glyphosate potássico, isolados, promoveram controle considerado ruim (inferior a 30\%) de ambas as espécies de trapoeraba, na dose de $720 \mathrm{~g} \mathrm{ha}^{-1}$. A eficiência de controle pelas misturas de herbicidas foi superior à das suas aplicações isoladas, com exceção do carfentrazone-ethyl em doses acima de $30 \mathrm{~g} \mathrm{ha}^{-1}$, as quais proporcionaram controles de C. benghalensis semelhantes às misturas. Apesar do razoável controle (de 71 a 80\%) para C. diffusa e do bom a excelente controle (acima de $81 \%$ ) para $C$. benghalensis, proporcionados pelas misturas de carfentrazone-ethyl com glyphosate e/ou glyphosate potássico, apenas uma aplicação não foi suficiente para o controle definitivo da Commelina spp., pois verificou-se para ambas as espécies, por meio da avaliação da BFPA, a reinfestação da área devido à recuperação das plantas, ou mesmo, no caso de C. benghalensis, a reinfestação a partir de sementes subterrâneas, que se tornaram viáveis após a morte da parte aérea provocada pelos herbicidas.
\end{abstract}

Palavras-chave: Commelina benghalensis, Commelina diffusa, controle, herbicidas.

\begin{abstract}
This research was conducted to evaluate the effectiveness of carfentrazone-ethyl, isolated and in mixture with to glyphosate or glyphosate-potassium salt, on controlling two dayflower species, Commelina diffusa and $\mathbf{C}$. benghalensis. These species were grown from stem segments in $12 \mathrm{~L}$ pots filled with soil, during 120 days. A complete randomized block design with four replicates was performed for each species. The treatments were carfentrazoneethyl $\left(0,10,20,30,40\right.$ and $\left.50 \mathrm{~g} \mathrm{ha}^{-1}\right)$, isolated and in mixture with glyphosate or glyphosatepotassium salt, these being applied at doses of $720 \mathrm{~g} \mathrm{ha}^{-1}$. The percentages of weed control and shoot fresh weight (SFW) were evaluated. C. diffusa was more tolerant to carfentrazone-ethyl alone or combined with both glyphosate and glyphosate-potassium salt than $\mathbf{C}$. benghalensis. Both glyphosate and glyphosate-potassium salt were inefficient (control below 30\%) when applied isolated, regardless of the species. The efficiency of controlling herbicide mixtures was greater than their single applications, except for the carfentrazone-ethyl in doses above $30 \mathrm{~g} \mathrm{ha} \mathrm{a}^{-1}$, with C. benghalensis, in which control was similar to the employed mixtures. Despite the reasonable control (from 71 to 80\%) for C. diffusa and the very good control (above 81\%) for C. benghalensis,
\end{abstract}

1 Recebido para publicação em 13/8/2001 e na forma revisada em 17/12/2001.

Parte da dissertação de tese de mestrado apresentada à Universidade Federal de Viçosa.

2 Mestrando, Departamento de Fitotecnia da Universidade Federal de Viçosa - UFV, 36571-000 Viçosa-MG, <ronchicp@yahoo.com.br>. ${ }^{3}$ Professor, Departamento de Fitotecnia da UFV. ${ }^{4}$ Bolsista de Iniciação Científica.

Planta Daninha, Viçosa-MG, v.20, n.1, p.103-113, 2002 
obtained with carfentrazone-ethyl + glyphosate or carfentrazone-ethyl + glyphosate-potassium salt mixtures, a sole application did not decisively control Commelina spp. In effect, recovery of plants as seen through SFW evaluation took place irrespective of the species; moreover, for $\mathbf{C}$. benghalensis, reinfestation from underground seeds that became viable after the shoot's death, due to herbicide application, was also found.

Key words: Commelina benghalensis, Commelina diffusa, control, herbicides.

\section{INTRODUÇÃO}

A família Commelinaceae apresenta entre 40 e 50 gêneros, com cerca de 700 espécies. As espécies infestantes que ocorrem no Brasil concentram-se em quatro gêneros; destes, o Commelina é o mais importante, de ampla distribuição no Brasil, sendo conhecido pelo nome comum de trapoeraba (Kissmann, 1997). Segundo esse autor, essas plantas daninhas revestem-se de grande importância, pois em áreas com suficiente umidade e temperatura há perenização por alastramentos sucessivos, e pedaços de ramos deixados no solo resistem ao estresse hídrico, suportando situações de baixa luminosidade por longo período até o aparecimento de condições ótimas para seu brotamento. Na cafeicultura, assim como em outras culturas perenes, a trapoeraba encontra condições muito favoráveis ao seu ótimo desenvolvimento, tornando-se uma das espécies de plantas daninhas mais importantes, causando forte interferência sobre as plantas de café, principalmente quando estas são jovens (Ronchi et al., 2001).

Além desses fatores, essa espécie merece atenção especial, pois é de difícil manejo químico, uma vez que apresenta tolerância aos herbicidas mais utilizados em lavouras cafeeiras para o controle não-seletivo de plantas daninhas: glyphosate e sulfosate, os quais são de uso generalizado em lavouras de café, pelo fato de serem eficientes sobre a maioria das espécies de plantas daninhas (Matiello, 1991). Além dessa característica intrínseca da trapoeraba (de tolerar determinado tratamento herbicida, mesmo sofrendo injúrias), a eliminação da competição com outras plantas daninhas pode ser fator responsável pela atual predominância dessa espécie nas lavouras (Ramos \& Durigan, 1996). A tolerância dessas espécies pode estar relacionada com a não-sensibilidade da enzima EPSPs aos herbicidas (Vargas et al., 1999) ou com características anatômicas e fisiológicas dessas plantas, como proposto por Santos et al. (2001).

A ineficiência do glyphosate no controle de trapoerabas é, também, relatada por Oliveira Jr. et al. (2000), Ronchi et al. (2001) e Santos et al. (2001). Estes últimos autores verificaram, ainda, tolerância diferencial entre a C. diffusa e a $C$. benghalensis, sendo aquela mais tolerante a esse herbicida.

Um dos herbicidas de uso generalizado em lavouras de café para o controle de trapoeraba é o 2,4-D. No entanto, este herbicida pode ser altamente tóxico ao homem, ao ambiente e também à lavoura, uma vez que é muito volátil (possui alta pressão de vapor) e de fácil deriva, sendo esta uma das razões de freqüentes injúrias às plantas de café, principalmente se jovens (Ronchi et al., 2001). Também, por apresentar pequena sorção (Vieira et al., 1999; Weber et al., 1965) e alta mobilidade no perfil do solo (Wilson \& Cheng, 1976; Hernandez \& Warren, 1950), mesmo não ocorrendo deriva, o 2,4-D pode atingir o sistema radicular do cafeeiro, ser absorvido e causar-lhe severa toxidez, quando aplicado em dose elevada.

Atualmente, devido à maior conscientização e exigência por parte da população e à maior pressão exercida pelos órgãos ambientais, as empresas têm investido na síntese de novas moléculas herbicidas que sejam eficientes agronomicamente, mas que causem baixo impacto ambiental. Uma dessas novas moléculas, recentemente desenvolvida, é o carfentrazoneethyl. Este herbicida apresenta efeito residual no solo muito curto, baixo potencial de deriva e baixa toxicidade (Oliveira Jr. et al., 2000). Além dessas características, o carfentrazoneethyl tem se mostrado altamente eficiente no controle de trapoeraba (Commelina spp.) e 
corda-de-viola (Ipomoea spp.), conforme pesquisas realizadas por Corrêa \& Borges (2000) e Oliveira Jr. et al. (2000).

O carfentrazone-ethyl pertence ao grupo químico das triazolinonas e tem como mecanismo de ação a inibição da protoporfirinogênio oxidase (PROTOX), que é a enzima envolvida na rota biossintética da clorofila (HRAC, 2001). Logo, nas plantas tratadas com este herbicida, ocorre acúmulo de protoporfirinogênio IX, que, na presença da luz, está envolvido na formação de oxigênio singleto, responsável pela peroxidação de membranas (Dayan et al., 1997). Isso causa rápida dessecação das espécies suscetíveis e a sintomatologia pode ser observada no mesmo dia da aplicação (Corrêa \& Borges, 2000).

Segundo Corrêa \& Borges (2000), o carfentrazone-ethyl pode apresentar efeito complementar quando misturado ao glyphosate, pois as plantas daninhas tolerantes ou não ao glyphosate, como Commelina benghalensis, mostram, já no primeiro dia após a aplicação, sintomas visuais de intoxicação e controle total aos 30 dias.

O objetivo deste trabalho foi avaliar a eficácia do carfentrazone-ethyl, isolado e em misturas com glyphosate e/ou glyphosate potássico, no controle de Commelina benghalensis e Commelina diffusa.

\section{MATERIAL E MÉTODOS}

O experimento foi conduzido em casa de vegetação, em vasos contendo $12,0 \mathrm{~L}$ de substrato, no período de junho a dezembro de 2000. O solo (LVAd) utilizado para enchimento dos vasos, de classe textural franco-argilo-arenosa, com $30 \mathrm{dag} \mathrm{kg}^{-1}$ de argila e 5,66 dag $\mathrm{kg}^{-1}$ de matéria orgânica, recebeu $1,0 \mathrm{~kg} \mathrm{~m}^{-3} \mathrm{de}_{2} \mathrm{O}_{5} \mathrm{e}$ $2,0 \mathrm{~kg} \mathrm{~m}^{-3}$ de calcário (PRNT=76\%), para suprir a necessidade de calagem (NC) de 3,0 t ha ${ }^{-1}$. Para cada vaso foram transplantados cinco segmentos de caule de $C$. benghalensis e de C. diffusa, com dois a três nós, coletadas na região cafeeira da Zona da Mata mineira, sendo cada espécie propagada em vasos separados.

Durante o período de condução do experimento foram realizadas duas fertilizações com $3,0,0,75$ e 3,0 g vaso-1 de N, $\mathrm{P}_{2} \mathrm{O}_{5}$ e $\mathrm{K}_{2} \mathrm{O}$, respectivamente, utilizando-se a fórmula 20-05-20, sendo a primeira aos dois meses após o transplantio dos acessos das trapoerabas e a segunda aos 30 dias após a aplicação dos tratamentos (DAT). A irrigação dos experimentos foi feita por microaspersão, sendo a lâmina de água ajustada em função da demanda das plantas e das condições ambientais diárias.

Cada espécie constituiu um experimento diferente, sendo conduzidos no delineamento em blocos casualizados com quatro repetições e 18 tratamentos (Tabela 1). Os herbicidas foram aplicados quatro meses após o transplantio dos acessos das trapoerabas, entre 7 e 10 horas da manhã, estando a temperatura e umidade relativa do ar em $23^{\circ} \mathrm{C}$ e $92 \%$, respectivamente. As plantas apresentavam-se completamente desenvolvidas, estando a C. diffusa com maior volume de massa verde e maior comprimento de ramos. Nessa ocasião foram coletadas amostras de solo nos vasos (bordadura), para caracterização química (Tabela 2), textural e da matéria orgânica do substrato no qual as plantas se desenvolviam.

Na aplicação dos herbicidas foi utilizado pulverizador costal de precisão, pressurizado a $\mathrm{CO}_{2}$, operando à pressão constante de $2,0 \mathrm{kgf} \mathrm{cm}^{-2}$, barra com dois bicos Turbo Teejet 110.03 , a uma altura de $30 \mathrm{~cm}$ das plantas, pulverizando-se o equivalente a $200 \mathrm{~L} \mathrm{ha}^{-1} \mathrm{de}$ calda. Ambas as espécies de trapoeraba receberam os tratamentos simultaneamente.

A eficácia dos tratamentos foi avaliada aos $7,14,21,28$ e 42 DAT, atribuindo-se notas percentuais de controle em relação à testemunha (sem aplicação) de cada bloco, sendo zero ausência de controle e cem, morte completa da planta. Para melhor interpretação dos resultados foram definidos níveis de controle (conceitos) em função de faixas de eficiência de controle, utilizando a escala proposta pela ALAM (1974), com algumas modificações: de 91 a 100\%, controle excelente; de 81 a 90\%, controle bom; de 71 a 80\%, controle razoável; de 51 a $70 \%$, controle insuficiente; e, quando inferior a 50\%, controle ruim.

Aos 60 DAT, foi feita a determinação da biomassa fresca da parte aérea (BFPA) das plantas de trapoeraba em cada parcela. Essa biomassa era formada por partes da planta que toleraram os tratamentos herbicidas, por novas brotações e, também, exclusivamente para 
C. benghalensis, por plantas originadas de sementes formadas no sistema radicular, em estruturas denominadas rizantógenos (Kissmann, 1997), que se tornaram viáveis após a morte da parte aérea, devido ao efeito dos herbicidas. Aos 100 DAT, ou 40 dias após a coleta da parte aérea, foi determinado o número de manifestações epígeas de $C$. benghalensis que emergiram nos vasos.

A verificação das pressuposições da análise de variância foi feita por meio da análise gráfica de resíduos (Neter et al., 1990), juntamente com o teste de $\mathrm{F}$ máximo, para a homogeneidade das variâncias. A análise de variância da porcentagem de controle foi realizada individualmente para cada época de avaliação, conforme Tabela 3, desdobrando-se os graus de liberdade de tratamento em grupos de contrastes ortogonais de interesse prático. A decomposição desses graus de liberdade foi feita de três formas diferentes, para obtenção dos contrastes de interesse, sendo mantida a ortogonalidade.

Tabela 1 - Tratamentos aplicados nas duas espécies de trapoeraba, em casa de vegetação. Viçosa-MG, 2000

\begin{tabular}{|c|c|c|c|c|}
\hline Tratamentos & \multicolumn{4}{|c|}{ Dose (g ha ${ }^{-1}$ i.a. $)$} \\
\hline 01. carfentrazone-ethyl ${ }^{1 /}+$ Assist $^{4 /}$ & 10 & $+0,5 \%$ & & \\
\hline 02. carfentrazone-ethyl + Assist & 20 & $+0,5 \%$ & & \\
\hline 03. carfentrazone-ethyl + Assist & 30 & $+0,5 \%$ & & \\
\hline 04. carfentrazone-ethyl + Assist & 40 & $+0,5 \%$ & & \\
\hline 05. carfentrazone-ethyl + Assist & 50 & $+\quad 0,5 \%$ & & \\
\hline 06. carfentrazone-ethyl + glyphosate ${ }^{-1}+$ Assist & 10 & +720 & + & $0,5 \%$ \\
\hline 07. carfentrazone-ethyl + glyphosate + Assist & 20 & +720 & + & $0,5 \%$ \\
\hline 08. carfentrazone-ethyl + glyphosate + Assist & 30 & +720 & + & $0,5 \%$ \\
\hline 09. carfentrazone-ethyl + glyphosate + Assist & 40 & +720 & + & $0,5 \%$ \\
\hline 10. carfentrazone-ethyl + glyphosate + Assist & 50 & +720 & + & $0,5 \%$ \\
\hline 11. carfentrazone-ethyl + glyphosate potássico ${ }^{-}+$Assist & 10 & +720 & + & $0,5 \%$ \\
\hline 12. carfentrazone-ethyl + glyphosate potássico + Assist & 20 & +720 & + & $0,5 \%$ \\
\hline 13. carfentrazone-ethyl + glyphosate potássico + Assist & 30 & +720 & + & $0,5 \%$ \\
\hline 14. carfentrazone-ethyl + glyphosate potássico + Assist & 40 & +720 & + & $0,5 \%$ \\
\hline 15. carfentrazone-ethyl + glyphosate potássico + Assist & 50 & +720 & + & $0,5 \%$ \\
\hline 16. glyphosate & 720 & & & \\
\hline 17. glyphosate potássico & 720 & & & \\
\hline 18. testemunha & - & & & \\
\hline
\end{tabular}

1/ Aurora $400 \mathrm{CE} ;{ }^{2 /}$ Sal de isopropilamina: marca comercial Roundup CS; ${ }^{3 /}$ Sal potássico: marca comercial Zapp Qi; ${ }^{4 /}$ Óleo mineral.

Tabela 2 - Resultados da análise química da amostra do solo coletada nos vasos no dia da aplicação dos tratamentos, em casa de vegetação. Viçosa-MG, $2000^{1 / 1}$

\begin{tabular}{|c|c|c|c|c|c|c|c|c|c|c|c|}
\hline \multicolumn{12}{|c|}{ Análise Química } \\
\hline \multirow[t]{2}{*}{$\mathrm{pH}$} & $\mathrm{P}$ & $\mathrm{K}$ & $\mathrm{Al}$ & $\mathrm{Ca}$ & $\mathrm{Mg}$ & $\mathrm{H}+\mathrm{Al}$ & SB & $\mathrm{t}$ & $\mathrm{T}$ & $\mathrm{V}$ & $\mathrm{m}$ \\
\hline & \multicolumn{2}{|c|}{$\left(\mathrm{mg} \mathrm{dm}^{-3}\right)$} & \multicolumn{7}{|c|}{$\left(\mathrm{cmol}_{\mathrm{c}} \mathrm{dm}^{-3}\right)$} & \multicolumn{2}{|c|}{$(\%)$} \\
\hline 6,4 & 171,6 & 172,0 & 0,0 & 9,29 & 1,56 & 1,65 & 11,29 & 11,29 & 12,94 & 87,2 & 0,0 \\
\hline
\end{tabular}

1/ Análises realizadas no Laboratório de Análises Físicas e Químicas de Solo do Departamento de Solos da Universidade Federal de Viçosa.

pH em água, $\mathrm{KCl}$ e $\mathrm{CaCl}_{2}$ - relação 1:2,5; $\mathrm{P}, \mathrm{Na}, \mathrm{K}, \mathrm{Fe}, \mathrm{Zn}, \mathrm{Mn}, \mathrm{Cu}$ - extrator Mehlich-1; $\mathrm{Al}, \mathrm{Ca}$ e $\mathrm{Mg}$ - extrator $\mathrm{KCl}-1 \mathrm{~mol} \mathrm{~L}{ }^{-1} ; \mathrm{H}+\mathrm{Al}$ extrator $\mathrm{Ca}(\mathrm{Oac})_{2} 0,5 \mathrm{~mol} \mathrm{~L}^{-1} \mathrm{pH} 7,0$. 
O tratamento testemunha foi excluído da análise de variância, para a variável 'controle', uma vez que foi utilizado como padrão de comparação para os outros tratamentos, sendo considerado 0\% de controle. Contudo, para a variável biomassa fresca da parte aérea (BFPA), fez-se ligeira modificação na tabela de análise de variância antes mencionada, incluindo-se o tratamento 'testemunha'. Em conseqüência disso, além dos contrastes apresentados na Tabela 3, foi adicionado o contraste $Y_{0}$, para testar todos os tratamentos com herbicidas versus a testemunha. Os demais contrastes permaneceram constantes, com pequena modificação nos contrastes $\mathrm{Y}_{5}$ e $\mathrm{Y}_{8}$ (contrastes sem interesse), para incluir a testemunha na análise de variância.

A relação da porcentagem de controle e da BFPA com as doses do carfentrazone-ethyl, tanto isoladas quanto nas misturas, foi analisada por regressão, testando-se os modelos linear e raiz quadrada. Tanto para a análise de variância quanto para a estimativa dos parâmetros da regressão do modelo utilizou-se o sistema estatístico SAEG (SAEG, 1997).

\section{RESULTADOS E DISCUSSÃO}

Constatou-se que todas as variáveis (\% de controle e biomassa fresca da parte aérea BFPA) atenderam as pressuposições da análise de variância. Assim, com base nos resumos das análises de variância da porcentagem de controle de C. diffusa e C. benghalensis e nas estimativas dos contrastes de interesse prático preestabelecidos (Tabela 3), foi possivel conhecer os efeitos dos tratamentos ou grupos de tratamentos estudados no controle dessas duas espécies de trapoeraba. A significância dos quadrados médios dos contrastes, para cada variável, indicou a existência de diferenças entre os grupos de tratamentos de cada contraste, enquanto a estimativa desses contrastes permitiu definir qual grupo de tratamentos proporcionou maior controle.

Desse modo, constatou-se que não houve diferença significativa ( $>0,05)$ para o controle de $C$. benghalensis quando foi aplicada a mistura de carfentrazone-ethyl + glyphosate ou carfentrazone-ethyl + glyphosate potássico. Observou-se que o aumento da dose de

Tabela 3 - Decomposição dos graus de liberdade de tratamentos em três grupos de contrastes ortogonais $\left(\right.$ Grupo A.Y.Y 1 , $\hat{\mathrm{Y}}_{2}$, $\hat{\mathrm{Y}}_{3}, \hat{\mathrm{Y}}_{4}$; Grupo B: $\hat{\mathrm{Y}}_{5}, \hat{\mathrm{Y}}_{6}, \hat{\mathrm{Y}}_{7} ;$ Grupo C: $\hat{\mathrm{Y}}_{8}, \hat{\mathrm{Y}}_{9}$ ) para o estudo dos efeitos dos grupos de tratamentos no controle de Commelina benghalensis e Commelina diffusa

\begin{tabular}{|c|c|c|}
\hline Grupo & Fontes de Variação & GL \\
\hline \multirow{6}{*}{ A } & $\begin{array}{l}\text { Bloco } \\
\text { Tratamentos }\end{array}$ & $\begin{array}{c}3 \\
(16)\end{array}$ \\
\hline & $\hat{\mathrm{Y}}_{1}=$ carfentrazone-ethyl vs. glyphosate e glyphosate potássico & 1 \\
\hline & $\widehat{\mathrm{Y}}_{2}=$ glyphosate vs. glyphosate potássico & 1 \\
\hline & $\widehat{\mathrm{Y}}_{3}=$ carfentrazone-ethyl isolado vs. carfentrazone-ethyl nas misturas & 1 \\
\hline & $\widehat{\mathrm{Y}}_{4}=$ carfentrazone + glyphosate vs. carfentrazone + glyphosate potássico & 1 \\
\hline & Outras comparações & 12 \\
\hline \multirow{4}{*}{$\mathrm{B}$} & $\hat{\mathrm{Y}}_{5}{ }^{*}=$ carfentrazone-ethyl isolado vs. resto (contraste sem interesse) & 1 \\
\hline & $\hat{\mathrm{Y}}_{6}=$ carfentrazone-ethyl + glyphosate vs. glyphosate & 1 \\
\hline & $\widehat{Y}_{7}=$ carfentrazone-ethyl + glyphosate potássico vs. glyphosate potássico & 1 \\
\hline & Outras comparações & 13 \\
\hline \multirow{5}{*}{$\mathrm{C}$} & $\hat{\mathrm{Y}}_{8}^{*}=$ herbicidas isolados vs. mistura (contraste sem interesse) & 1 \\
\hline & $\widehat{\mathrm{Y}}_{9}=$ carfentrazone-ethyl isolado vs. glyphosate e glyphosate potássico & 1 \\
\hline & Outras comparações & 14 \\
\hline & Resíduo & 48 \\
\hline & Total & 67 \\
\hline
\end{tabular}

* Não há interesse prático nos contrastes $\hat{\mathrm{Y}}_{5}$ e $\hat{\mathrm{Y}}_{8}$. Eles foram montados apenas para a obtenção dos contrastes ortogonais de interesse, $\widehat{\mathrm{Y}}_{6}$ $\hat{\mathrm{Y}}_{7}$ e $\hat{\mathrm{Y}}_{9}$. 
carfentrazone-ethyl na mistura, principalmente com glyphosate potássico, proporcionou maior controle de $C$. benghalensis. Esse efeito pode ser visualizado, por exemplo, na avaliação realizada aos 28 DAT (Figura 1-H), quando se obteve 95\% de controle com a dose de $50 \mathrm{~g} \mathrm{ha}^{-1}$ contra $79 \%$ com a dose de $10 \mathrm{~g} \mathrm{ha}^{-1}$. Já quando misturado com glyphosate o controle permaneceu em torno de $80 \%$, a partir dos 28 DAT (Figura 1-H e J), independentemente da dose de carfentrazone-ethyl na mistura.

A mistura de carfentrazone-ethyl com glyphosate potássico (Zapp Qi) foi superior (p<0,05), no controle de $C$. diffusa, à mistura com glyphosate, com exceção da avaliação realizada aos 28 DAT. Até os 21 DAT (Figura 1-A, C e E), a mistura de carfentrazone-ethyl com glyphosate potássico foi superior à mistura com glyphosate quando o carfentrazone-ethyl estava presente em doses superiores a $20 \mathrm{~g} \mathrm{ha}^{-1}$. Todavia, aos 42 DAT (Figura 1-I), a mistura de carfentrazone-ethyl com glyphosate potássico foi superior à outra, independentemente da dose de carfentrazone-ethyl utilizada.

As aplicações isoladas de glyphosate ou glyphosate potássico, na dose de $720 \mathrm{~g} \mathrm{ha}^{-1}$, proporcionaram controle inferior a $30 \%$ de ambas as espécies de trapoeraba (Figura 1-A a J), não se observando diferenças significativas $(p>0,05)$ entre esses tratamentos. Entretanto, Santos et al. (2001), conduzindo experimento em condições semicontroladas, obtiveram controle excelente $(94,5 \%)$ para $C$. benghalensis, com doses iguais ou superiores a $720 \mathrm{~g} \mathrm{ha}^{-1}$, a partir dos 25 DAT.

De acordo com os resultados apresentados no parágrafo anterior, não se observou tolerância diferencial entre as espécies de trapoeraba para a dose de $720 \mathrm{~g}^{\text {ha }}{ }^{-1}$ de ambos os herbicidas inibidores da EPSPs avaliados, diferindo, assim, dos resultados de Santos et al. (2001).

Contudo, C. diffusa (Figura 1-A, C, E, G e I) mostrou-se mais tolerante ao carfentrazoneethyl e às misturas deste ao glyphosate e ao glyphosate potássico (controle inferior a 80\%) do que $C$. benghalensis (controle superior a $81 \%$ - Figura 1-B, D, F, H e J).

A mistura do carfentrazone-ethyl tanto ao glyphosate quanto ao glyphosate potássico foi superior no controle das duas espécies de trapoerabas, quando comparadas com as aplicações desses mesmos herbicidas isoladamente. Oliveira Jr. et al. (2000) verificaram que a mistura de carfentrazone-ethyl (30 e $50 \mathrm{~g} \mathrm{ha}^{-1}$ ) com glyphosate ( $960 \mathrm{~g} \mathrm{ha}^{-1}$ ) proporcionou niveis de controle de $C$. benghalensis e Ipomoea grandifolia melhores do que aqueles observados paras esses herbicidas utilizados isoladamente, sugerindo ser essa mistura uma opção para dessecações em áreas de semeadura direta, uma vez que não deixam resíduos no solo para culturas como soja e milho. Essa complementação apresentada pela mistura, como também já observado por Corrêa \& Borges (2000), tem sido um dos pontos mais explorados pelas pesquisas com esses herbicidas, como solução para o manejo da trapoeraba, seja em áreas de semeadura direta (dessecações) ou em lavouras cafeeiras, visto que esta espécie daninha está entre aquelas de maior importância na cafeicultura.

Além de a mistura proporcionar melhor controle, os sintomas de sua toxidez na trapoeraba apareceram rapidamente, no dia seguinte à aplicação. Resultados semelhantes foram observados por Corrêa \& Borges (2000) e Oliveira Jr. et al. (2000). A rapidez no aparecimento dos sintomas de toxidez na trapoeraba é proporcionada pelo carfentrazone-ethyl, o qual, segundo a HRAC (2001), é inibidor da enzima protoporfirinogênio oxidase (PROTOX), envolvida na rota biossintética da clorofila.

A presença do carfentrazone-ethyl na calda aplicada proporcionou controle maior das trapoerabas do que a aplicação do glyphosate ou glyphosate potássico isoladamente. Para C. benghalensis, essa superioridade ocorreu não somente devido à mistura, mas também devido à aplicação do carfentrazone-ethyl isolado, uma vez que, ao se comparar o controle proporcionado pelo carfentrazone-ethyl com o controle proporcionado pelo glyphosate e glyphosate potássico, constatou-se que aquele foi superior. Isso também foi verdadeiro para C. diffusa, nas avaliações feitas aos 7 e 14 DAT. Todavia, aos 21 e 28 DAT, não houve diferenças significativas ( $p>0,05)$ entre os tratamentos; aos 42 DAT, a situação inverteu-se, e o glyphosate e o glyphosate potássico foram superiores ao carfentrazone-ethyl isolado. Isso ocorreu pelo fato de inicialmente o carfentrazone-ethyl ter causado alta toxidez em $C$. diffusa, porém houve rápida recuperação das plantas (Figura 1-A, C, E, G e I). 

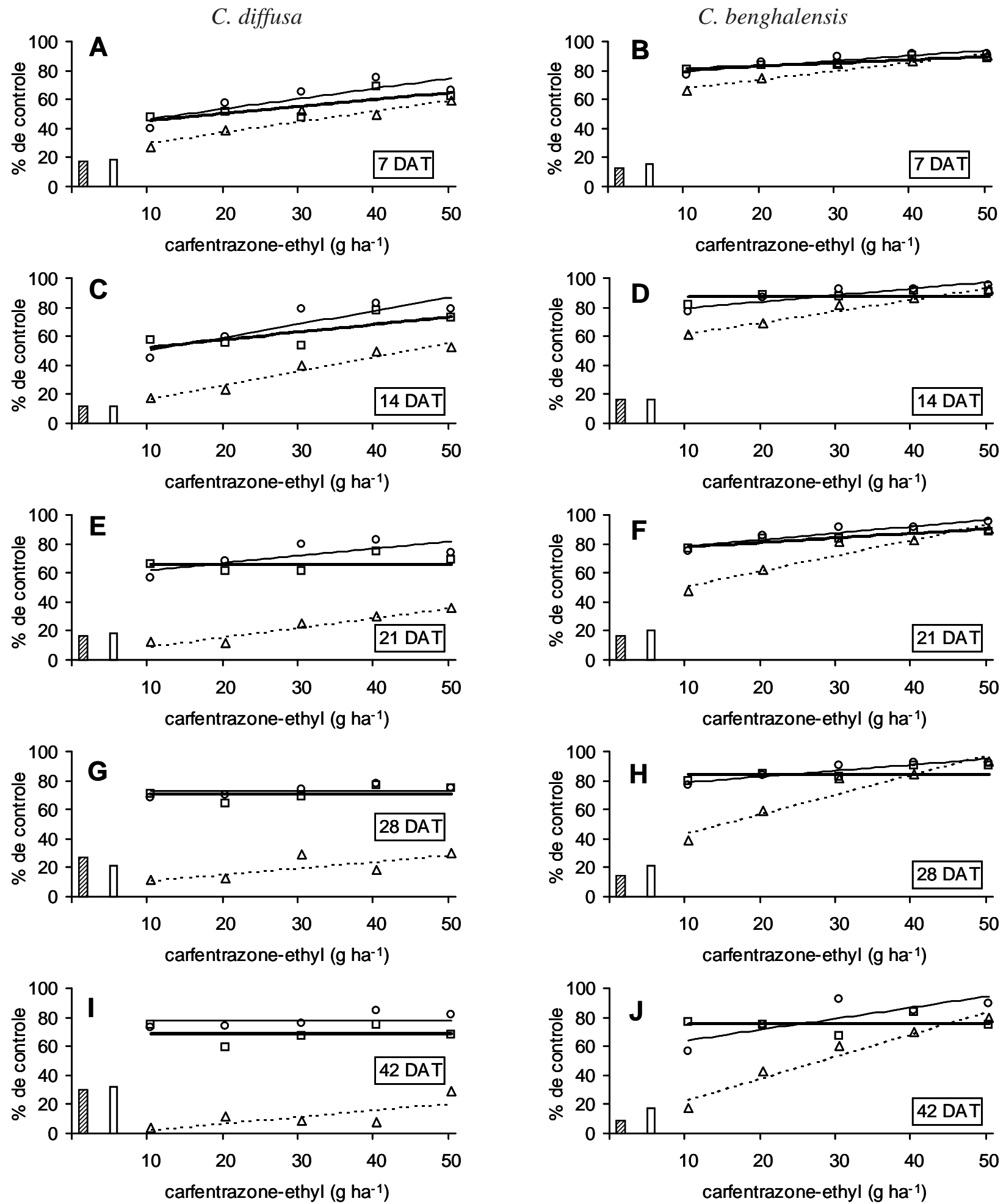

212101 glyphosate glyphosate K ....... carfentrazone — carf. + gly $\longrightarrow$ carf.+ gli K

Figura 1 - Efeito do glyphosate $\left(720 \mathrm{~g} \mathrm{ha}^{-1}\right)$, glyphosate potássico $\left(720 \mathrm{~g} \mathrm{ha}^{-1}\right)$, carfentrazone-ethyl $\left(10\right.$ a $\left.50 \mathrm{~g} \mathrm{ha}^{-1}\right)$, carfentrazoneethyl + glyphosate $\left(10\right.$ a $\left.50+720 \mathrm{~g} \mathrm{ha}^{-1}\right)$ e carfentrazone-ethyl + glyphosate potássico $\left(10 \mathrm{a} 50+720 \mathrm{~g}^{-1}\right)$ no controle de C. diffusa e C. benghalensis aos 7, 14, 21, 28 e 42 DAT. 
Na Figura 1, em quase todas as épocas de avaliação, observou-se que o aumento da dose de carfentrazone-ethyl, no intervalo de 10 a $50 \mathrm{~g} \mathrm{ha}^{-1}$, promoveu aumento no controle das duas espécies de trapoeraba, principalmente quando esse herbicida foi aplicado isoladamente. Nessa condição, para C. diffusa, a maior eficácia de controle foi obtida aos 7 DAT (Figura 1-A) com a maior dose de carfentrazone-ethyl $\left(50 \mathrm{~g} \mathrm{ha}^{-1}\right)$. Contudo, nas épocas subseqüentes, tendo em vista a recuperação das plantas, a porcentagem de controle foi diminuindo (Figura 1-A, C, E, G e I), chegando a valores próximos de zero para a dose de $10 \mathrm{~g} \mathrm{ha}^{-1}$ e inferiores a 30\% para a dose de $50 \mathrm{~g} \mathrm{ha}^{-1}$ (Figura 1-I).

Para C. benghalensis, que se mostrou mais sensivel ao carfentrazone-ethyl, observou-se aos 7 DAT (Figura 1-B), considerando-se os valores extremos das doses, controle variando de 65 a $85 \%$ e, aos 42 DAT (Figura 1-J), eficácia de controle variando de 20 a $85 \%$. Isso indica que até a época da última avaliação não houve recuperação das trapoerabas quando foram aplicados $50 \mathrm{~g} \mathrm{ha}^{-1}$ de carfentrazone-ethyl. Essas diferenças de controle, nas várias épocas, podem ser visualizadas na Figura 1, ou verificadas simplesmente observando-se o comportamento dos parâmetros das equações ajustadas (modelos de regressão linear simples: $\mathrm{Y}=\mathrm{a}+$ bx) (Tabela 4): diminuição no valor do intercepto e aumento no valor do coeficiente angular das retas, à medida que foram feitas avaliações mais tardias.

Com relação à avaliação da biomassa fresca da parte aérea (BFPA) realizada aos 60 DAT, observou-se que todos os tratamentos contribuíram significativamente $(\mathrm{p}<0,01)$ para a redução da BFPA, comparativamente à testemunha (Figura 2-A e B). Devido à maior tolerância (ou menor controle) de C. diffusa aos herbicidas utilizados e à rápida recuperação das plantas, com lançamento de novas brotações, essa espécie (Figura 2-A) apresentou valores maiores de BFPA (Tabela 4), comparativamente a $C$. benghalensis (Figura 2-B).

A maior redução $(\mathrm{p}<0,01)$ na produção de BFPA de $C$. diffusa foi proporcionada pela mistura de carfentrazone-ethyl + glyphosate potássico, seguida da mistura de carfentrazone-ethyl + glyphosate e, por último, pelo carfentrazoneethyl isolado (Figura 2-A). Não houve diferenças significativas $(p>0,05)$ entre glyphosate e glyphosate potássico isolados e entre glyphosate isolado e sua mistura ao carfentrazone-ethyl. No entanto, foi significativa $(p<0,05)$ a maior redução da BFPA de $C$. diffusa pela mistura do carfentrazone-ethyl + glyphosate potássico, comparada ao glyphosate potássico isolado.

Para C. benghalensis, a partir dos 28 DAT e, principalmente, aos 42 DAT, observou-se o início da reinfestação dos vasos. Esta não ocorreu somente a partir de brotações de partes vegetativas que toleram os tratamentos herbicidas, mas principalmente devido à germinação e emergência de plantas originadas de sementes formadas em estruturas subterrâneas (rizomas), que se tornaram viáveis com a morte da parte aérea. Essas novas plantas e brotações apresentaram crescimento rápido e alto vigor vegetativo. Dessa forma, a BFPA de C. benghalensis, determinada aos 60 DAT, representava a reinfestação que ocorreu naqueles tratamentos (misturas de carfentrazoneethyl ao glyphosate e ao glyphosate potássico e aplicação isolada do carfentrazone-ethyl em doses superiores a $30 \mathrm{~g} \mathrm{ha}^{-1}$ ) onde o controle havia sido bom e excelente. Naqueles vasos em que o controle foi ruim (por exemplo, pela aplicação de glyphosate ou do glyphosate potássico) e na testemunha, a BFPA era aquela remanescente desde o início do experimento, sem reinfestação. Logo, pela Figura 2-B observa-se que a reinfestação foi ligeiramente maior $(p<0,05)$ para carfentrazone-ethyl + glyphosate do que para carfentrazone-ethyl + glyphosate potássico. O carfentrazone-ethyl proporcionou comportamento variado em função das doses: baixas doses não controlaram totalmente a parte aérea e contribuíram para o maior valor da BFPA; para doses altas, observou-se o contrário (Figura 2-B). O maior valor de BFPA para o glyphosate isolado, em relação ao glyphosate potássico (Figura 2-B), indica ligeira superioridade $(\mathrm{p}<0,05)$ do glyphosate potássico no controle de $C$. benghalensis.

Pode-se inferir desses resultados que, $a$ priori, muitos dos tratamentos utilizados foram eficientes no controle de $C$. benghalensis. Contudo, nenhum deles proporcionou niveis de controle de $100 \%$. Transportando esses resultados para condições reais, no campo, mesmo que esses tratamentos herbicidas proporcionassem controle total da parte aérea dessa espécie daninha, seria inevitável a reinfestação da área, visto que, com a morte da parte aérea, 
as sementes subterrâneas seriam estimuladas a germinar, caso a planta tivesse atingido o estádio reprodutivo.

Germinação de sementes subterrâneas semelhante àquela que causou a infestação dos vasos (resultados já discutidos) foi verificada novamente aos 40 dias após a eliminação da parte aérea de $C$. benghalensis, quando se fez a contagem do número de plantas emergidas em cada vaso (Tabela 5). Para aqueles tratamentos (testemunha, glyphosate e glyphosate potássico), em cujos vasos ainda não havia ocorrido germinação de sementes subterrâneas, uma vez que a parte aérea não havia morrido, observou-se a germinação (média desses tratamentos) de maior número de plantas (superior a 10 plantas vaso ${ }^{-1}$ ), devido ao provável maior banco de sementes viáveis formadas nos rizomas subterrâneos. Nos demais tratamentos (Tabela 5), ocorreu, em média, menor número de plantas emergidas (inferior a 7 plantas vaso ${ }^{-1}$ ), pois nesses vasos já haviam ocorrido germinações após a morte da parte aérea causada pelos herbicidas.

Tabela 4 - Equações relacionando porcentagem de controle de $C$. diffusa e $C$. benghalensis em função de doses crescentes de carfentrazone-ethyl, aplicadas isoladamente (C) ou em mistura com $720 \mathrm{~g} \mathrm{ha}^{-1}$ de glyphosate (C+G) ou glyphosate potássico $(\mathrm{C}+\mathrm{CG})$, em várias épocas de avaliação, e também equações relacionando a BFPA, aos 60 DAT, em função dessas mesmas variáveis regressoras. Viçosa-MG, 2000

\begin{tabular}{|c|c|c|c|c|}
\hline \multirow{2}{*}{ Variável } & \multicolumn{2}{|c|}{ C. diffusa } & \multicolumn{2}{|c|}{ C. benghalensis } \\
\hline & Equações & $\mathrm{R}^{2}$ & Equações & $\mathrm{R}^{2}$ \\
\hline \multicolumn{5}{|c|}{ 7 DAT } \\
\hline $\mathrm{C}$ & $\hat{\mathrm{Y}}=23,375+0,7375^{* *} \mathrm{X}$ & $0,885^{* *}$ & $\hat{\mathrm{Y}}=62,725+0,5875^{* *} \mathrm{X}$ & $0,987 * *$ \\
\hline $\mathrm{C}+\mathrm{G}$ & $\hat{\mathrm{Y}}=41,250+0,4750 * * \mathrm{X}$ & $0,609 * *$ & $\hat{\mathrm{Y}}=79,225+0,2075^{* *} \mathrm{X}$ & $0,817 * *$ \\
\hline $\mathrm{C}+\mathrm{GK}$ & $\hat{Y}=39,750+0,7000 * * X$ & $0,708^{* *}$ & $\hat{Y}=75,930+0,3600 * * X$ & $0,818^{* *}$ \\
\hline \multicolumn{5}{|c|}{$14 \mathrm{DAT}$} \\
\hline $\mathrm{C}$ & $\hat{\mathrm{Y}}=7,875+0,9625 * * \mathrm{X}$ & $0,951^{* *}$ & $\hat{\mathrm{Y}}=54,425+0,7875^{* *} \mathrm{X}$ & $0,975^{* *}$ \\
\hline $\mathrm{C}+\mathrm{G}$ & $\hat{\mathrm{Y}}=47,500+0,5250 * * \mathrm{X}$ & $0,574 * *$ & $\hat{\mathrm{Y}}=\overline{\mathrm{Y}}=87,55$ & - \\
\hline $\mathrm{C}+\mathrm{GK}$ & $\hat{Y}=41,370+0,9100 * * X$ & $0,791 *$ & $\hat{Y}=74,940+0,4500 * * X$ & $0,868 * *$ \\
\hline \multicolumn{5}{|c|}{ 21DAT } \\
\hline $\mathrm{C}$ & $\hat{\mathrm{Y}}=3,125+0,6625^{* *} \mathrm{X}$ & $0,920^{* *}$ & $\hat{\mathrm{Y}}=41,000+1,0650 * * \mathrm{X}$ & $0,919 * *$ \\
\hline $\mathrm{C}+\mathrm{G}$ & $\hat{\mathrm{Y}}=\overline{\mathrm{Y}}=66,50$ & - & $\hat{\mathrm{Y}}=75,100+0,3100 * \mathrm{X}$ & $0,834 *$ \\
\hline $\mathrm{C}+\mathrm{GK}$ & $\hat{\mathrm{Y}}=57,000+0,5000 * * \mathrm{X}$ & $0,561 * *$ & $\hat{Y}=73,780+0,4600 * * X$ & $0,854 * *$ \\
\hline \multicolumn{5}{|c|}{$28 \mathrm{DAT}$} \\
\hline $\mathrm{C}$ & $\hat{\mathrm{Y}}=7,125+0,4375^{* *} \mathrm{X}$ & $0,616^{* *}$ & $\hat{\mathrm{Y}}=30,775+1,3525 * * \mathrm{X}$ & $0,922 * *$ \\
\hline $\mathrm{C}+\mathrm{G}$ & $\hat{\mathrm{Y}}=\overline{\mathrm{Y}}=71,00$ & - & $\hat{\mathrm{Y}}=\overline{\mathrm{Y}}=84,50$ & - \\
\hline $\mathrm{C}+\mathrm{GK}$ & $\hat{\mathrm{Y}}=\overline{\mathrm{Y}}=72,65$ & - & $\hat{Y}=74,792+0,4058 * * X$ & $0,858^{* *}$ \\
\hline \multicolumn{5}{|c|}{$42 \mathrm{DAT}$} \\
\hline $\mathrm{C}$ & $\hat{\mathrm{Y}}=-1,875+0,4625 * * \mathrm{X}$ & $0,563^{* *}$ & $\hat{\mathrm{Y}}=8,250+1,525^{* * X}$ & $0,956^{* * *}$ \\
\hline $\mathrm{C}+\mathrm{G}$ & $\hat{\mathrm{Y}}=\overline{\mathrm{Y}}=68,65$ & - & $\hat{\mathrm{Y}}=\overline{\mathrm{Y}}=75,4$ & - \\
\hline $\mathrm{C}+\mathrm{GK}$ & $\hat{\mathrm{Y}}=\overline{\mathrm{Y}}=77,40$ & - & $\hat{\mathrm{Y}}=56,610+0,760 * * \mathrm{X}$ & $0,690^{* *}$ \\
\hline \multicolumn{5}{|c|}{ BFPA - 60 DAT } \\
\hline $\mathrm{C}$ & $\hat{\mathrm{Y}}=\overline{\mathrm{Y}}=795,00$ & - & $\hat{\mathrm{Y}}=749,000-11,75^{* * X}$ & $0,930 * *$ \\
\hline $\mathrm{C}+\mathrm{G}$ & $\hat{\mathrm{Y}}=\overline{\mathrm{Y}}=472,25$ & - & $\hat{\mathrm{Y}}=\overline{\mathrm{Y}}=373,00$ & - \\
\hline $\mathrm{C}+\mathrm{GK}$ & $\hat{\mathrm{Y}}=\overline{\mathrm{Y}}=294,75$ & - & $\hat{\mathrm{Y}}=\overline{\mathrm{Y}}=244,85$ & - \\
\hline
\end{tabular}

*, ** Significativo a 5 e $1 \%$ de probabilidade, respectivamente, pelo teste $\mathrm{F}$. 

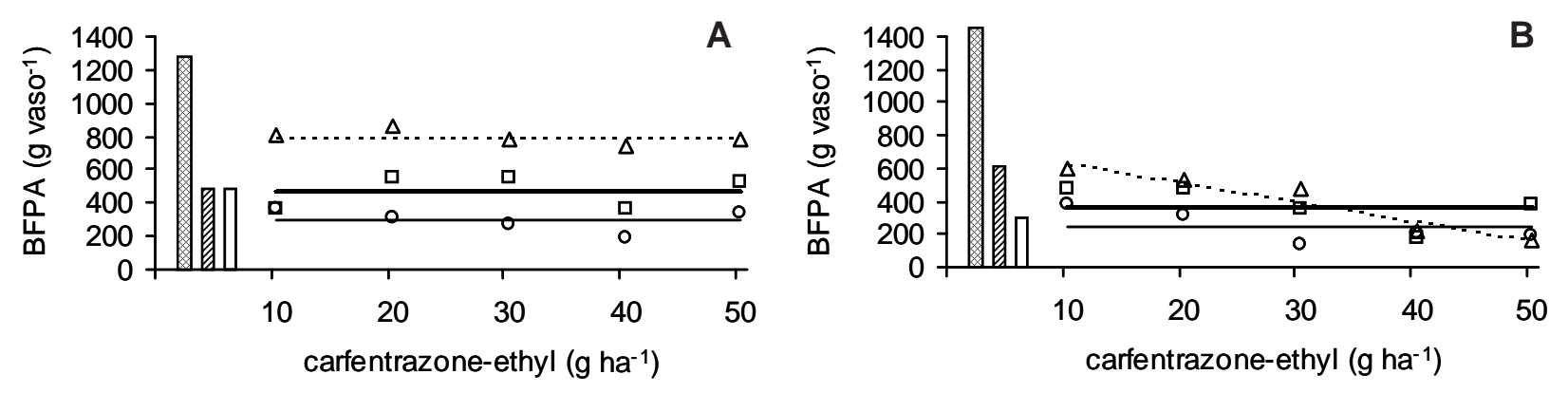

testemunha $\square$ glyphosate K - - - $\Delta-$ - carfentrazone glyphosate $\square$ carf. + gly $\longrightarrow$ carf.+ gli K

Figura 2 - Biomassa fresca da parte aérea de C. diffusa (A) e C. benghalensis (B), aos 60 DAT, em função de glyphosate $\left(720 \mathrm{~g} \mathrm{ha}^{-1}\right)$, glyphosate potássico $\left(720 \mathrm{~g} \mathrm{ha}^{-1}\right)$, carfentrazone-ethyl $\left(10 \mathrm{a} 50 \mathrm{~g} \mathrm{ha}^{-1}\right)$, carfentrazone-ethyl + glyphosate $(10 \mathrm{a}$ $\left.50+720 \mathrm{~g} \mathrm{ha}^{-1}\right)$ e carfentrazone-ethyl + glyphosate potássico $\left(10\right.$ a $\left.50+720 \mathrm{~g} \mathrm{ha}^{-1}\right)$.

Tabela 5 - Médias do número de plantas de C. benghalensis emergidas nos vasos, aos 40 dias após a eliminação da parte aérea das "plantas-mãe", em função de grupos de tratamentos (médias de quatro blocos) e seus respectivos valores extrapolados para $1,0 \mathrm{~m}^{2}$ de solo. Viçosa-MG, 2000

\begin{tabular}{|c|c|c|}
\hline Grupos de tratamentos & Plantas emergidas/vaso (área ${ }_{\text {vaso }}=0,0573 \mathrm{~m}^{2}$ ) & Plantas $\mathrm{m}^{-2}$ de solo \\
\hline Testemunha & 11,00 & 192 \\
\hline Glyphosate & 16,00 & 279 \\
\hline Glyphosate potássico & 9,75 & 170 \\
\hline Carfentrazone-ethyl ${ }^{1 /}$ & 6,85 & 120 \\
\hline$(\text { carfentrazone-ethyl }+ \text { glyphosate })^{1^{\prime}}$ & 6,90 & 120 \\
\hline$(\text { carfentrazone-ethyl }+ \text { glyphosate potássico })^{\underline{1}}$ & 6,25 & 110 \\
\hline
\end{tabular}

${ }^{1 /}$ Refere-se à média de cinco tratamentos, ou seja, às doses de $10 \mathrm{a} 50 \mathrm{~g} \mathrm{ha}^{-1}$ de carfentrazone-ethyl, isolado e em misturas.

Pelos números apresentados na Tabela 5 , considerando apenas a testemunha, observa-se o alto potencial de reinfestação do solo (192 plantas $\mathrm{m}^{-2}$ ) por $C$. benghalensis, concordando com os resultados de Santos et al. (2001), que, durante aproximadamente os 60 dias que sucederam a morte da parte aérea de $C$. benghalensis, causada por doses diferenciadas de glyphosate, observaram a emergência média de 993 plantas $\mathrm{m}^{-2}$, em ambiente desprotegido, em cultivos em vasos.

Assim, é imprescindível a identificação da espécie presente na lavoura para se fazer o manejo adequado da trapoeraba. Este deverá ser realizado antes que essa planta daninha atinja o florescimento, a fim de evitar que sementes subterrâneas sejam formadas. São necessárias reaplicações dos herbicidas para garantir o controle por maior tempo, uma vez que a capacidade de rebrota e reinfestação do solo por essas plantas é grande. Faz-se mister pesquisas sobre o manejo do banco de sementes subterrâneas de $C$. benghalensis, talvez com aplicações de herbicidas ou misturas de herbicidas que, além da ação em pós-emergência, apresentem efeito residual no solo.

\section{LITERATURA CITADA}

ASOCIACIÓN LATINOAMERICANA DE MALEZAS ALAM. Recomendaciones sobre unificación de los sistemas de evaluacion en ensyos de control de malezas. ALAM, v.1, n. 1, p. 35-38, 1974.

CORRÊA, L. E. A.; BORGES, A. Glyphosate + carfentrazone: controle de ervas problemas. In: CONGRESSO BRASILEIRO DA CIÊNCIA DAS PLANTAS DANINHAS, 22, 2000, Foz do Iguaçu. Resumos... Londrina: Sociedade Brasileira da Ciência das Plantas Daninhas, 2000. p. 463.

DAYAN, F. E. et al. Selectivity and mode of action of carfentrazone-ethyl, a novel phenyl triazolinone herbicide. Pestic. Sci., v. 51, p. 65-73, 1997. 
HERBICIDE RESISTANCE ACTION COMMITEE HRAC. Classification of herbicides according to mode of action. [29/01/2001]. (http://www.plantprotection.org/hrac/ moa2001.htm.).

HERNANDEZ, T. P.; WARREN, G. F. Some factors affecting the rate of inactivation and leaching of 2,4-D in differente soils. Proc. Am. Soc. Hortic. Sci., v. 56, p. 287 293, 1950.

KISSMANN, K. G. Plantas infestantes e nocivas. 2.ed. São Paulo: BASF Brasileira, 1997. T.1. 825 p.

MATIELlO, J. B. O café: do cultivo ao consumo. São Paulo: Globo, 1991. 320 p.

NETER, J.; WASSERMAN, V.; KUTNER, M. H. Applied linear statistical models: regression, analysis of variance and experimental designs. Homewood: Richard A. Irwin, 1990. $842 \mathrm{p}$.

OLIVEIRA Jr., R.S. et al. Carfentrazone: novo herbicida para o manejo de Ipomoea grandifolia e Commelina benghalensis em áreas de semeadura direta de soja e milho. In: CONGRESSO BRASILEIRO DA CIÊNCIA DAS PLANTAS DANINHAS, 22, 2000, Foz do Iguaçu. Resumos... Londrina: Sociedade Brasileira da Ciência das Plantas Daninhas, 2000. p. 440.

RAMOS, H. H.; DURIGAN, J. C. Avaliação da eficiência da mistura pronta de glyphosate +2,4-D no controle da Commelina virginica L. em citrus. Planta Daninha, v. 14, n. 1, p. 33-41, 1996.
RONCHI, C. P.; SILVA, A. A.; FERREIRA, L. R. Manejo de plantas daninhas em lavouras de café. Viçosa, MG: Suprema, 2001. 94 p.

SISTEMA DE ANÁLISES ESTATÍSTICAS E GENÉTICAS - SAEG. Versão 7.1. Viçosa, MG: Fundação Arthur Bernardes, 1997.

SANTOS, I. C. et al. Eficiência de glyphosate no controle de Commelina benghalensis e Commelina diffusa. Planta Daninha, v. 19, n. 1, p. 135-143, 2001.

VARGAS, L. et al. Resistência de plantas daninhas a herbicidas. Viçosa, MG: Jard, 1999. 131 p.

VIEIRA, E. M. et al. Estudo da adsorção/dessorção do ácido 2,4 diclorofenoxiacético (2,4-D) em solo na ausência e presença de matéria orgânica. Química Nova, v. 23, n. 3, p. 305-308,1999.

WEBER, J. B.; PERRY, P. W.; UPCHURCH, R. P. The influence of temperature and time on the adsoption of paraquat, diquat, 2,4-D and prometone by clays, charcoal, and an anion-exchange resin. Proc. Soil Sci. Soc. Am., v. 29, p. $678-688,1965$.

WILSON Jr., R. G.; CHENG, H. H. Breakdown and movement of 2,4-D in the soil under field conditions. Weed Sci., v. 24, n. 5, p. 441-466, 1976. 